\title{
Calidad microbiológica del agua de un área agrícola-ganadera del centro sur de Chile y su posible implicancia en la salud humana
}

\author{
Eduardo Valenzuela, Roberto Godoy, Leonardo Almonacid y Mónica Barrientos
}

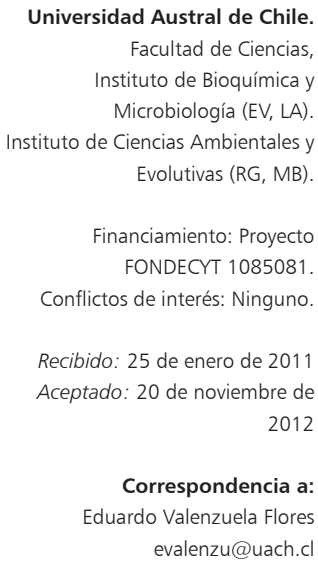

Universidad Austral de Chile.

Facultad de Ciencias,

Instituto de Bioquímica y

Microbiología (EV, LA).

Instituto de Ciencias Ambientales y

Evolutivas (RG, MB)

Financiamiento: Proyecto FONDECYT 1085081 Conflictos de interés: Ninguno.

Recibido: 25 de enero de 2011 Aceptado: 20 de noviembre de

Correspondencia a: Eduardo Valenzuela Flores evalenzu@uach.c

\section{Microbiological quality of water in livestock area of southern Chile and its possible implications on human health}

Introduction: A significant proportion of water for human consumption has an underground origin becoming $80 \%$ in Chilean rural areas where profound wells represent the only search of water for human and animal beverage. Aim: To study the microbiological quality of water from agricultural land for livestock production in the province of Valdivia $\left(40^{\circ} \mathrm{S}\right)$, Chile and its potential impact on human health. Material and Method: Water samples were collected monthly (2008 - 2009), at the entrance and exit of a water stream running through the field and in well water used for human and animal consumption. The total coliform (Tc) and Escherichia coli were determined by the confirmatory method Quanti-Tray together with other physicochemical assessments in the water and climatic variables. Results: In samples from the stream water and wells, the Most Probable Number of Tc and E. coli exceeded the standard Chilean Norm of Water Quality (NCh 409/1) for human consumption. Conclusion: These results show the need to regulate the environmental impact of farming and cattle production and to monitor the drinking water to meet the minimum standards of health protection.

Key words: Well water, microbiological water quality, coliforms, human health.

Palabras clave: Agua de pozos, calidad microbiológica del agua, coliformes, salud humana.

\section{Introducción}

L a química del agua de precipitación en áreas montañosas del sur de Chile, refleja una de las aproximaciones más cercanas a la condición pre-industrial ${ }^{1}$. Sin embargo, el aumento sostenido de la actividad agrícola y ganadera, con aplicación de purines, puede influir de forma significativa tanto en la química de la atmósfera, suelo, como en el agua de escorrentía superficial y napas freáticas ${ }^{2}$. El agua en sitios agropecuarios representa una gran parte del volumen total de los desechos generados por actividades lecheras y ganaderas, $46 \%$ del total de los residuos corresponden a agua de precipitación, 29\% a agua utilizada en faenas de limpieza y sólo $25 \%$ corresponde a fecas y orina ${ }^{3}$, aspectos que tienen especial relevancia para la salud en zonas de elevada precipitación.

En Chile, $40 \%$ del agua para consumo humano corresponde a agua subterránea, alcanzando un $80 \%$ en áreas rurales, donde los pozos profundos son la única fuente de agua de bebida para consumo humano y animal ${ }^{4}$. La calidad del agua puede variar con gran rapidez, por ejemplo, la lluvia puede hacer aumentar la contaminación microbiana en aguas de origen y son frecuentes los brotes de enfermedades después de periodos de precipitaciones, circunstancia que debe tenerse en consideración al interpretar los resultados de los análisis ${ }^{5}$.

Para evaluar la calidad del agua se usan parámetros físico-químicos y biológicos; en este último caso, principalmente las bacterias coliformes totales $(\mathrm{Ct}) \mathrm{y}$ Escherichia coli son usadas como bio-indicadores de contaminación fecal humana o de otros animales de sangre caliente, incluyéndose en estándares de calidad del agua de distintas regiones del mundo ${ }^{6}$. Se considera que niveles bajos de $\mathrm{Ct}$ son un buen indicador de ausencia de microorganismos patógenos, mientras que aguas altamente contaminadas con bacterias entéricas $(E$. coli), podrían ser una fuente potencial de contagio de enfermedades para el ser humano y el ganado, debido a la presencia de microorganismos patógenos (Salmonella, Shigella, Vibrio, virus de la hepatitis A y E, Entamoeba, etc.), capaces de causar enfermedades a través del agua de bebida ${ }^{7,8}$. Al respecto, en el año 2001 el consumo de agua dulce y de productos marinos costeros provenientes de áreas contaminadas produjo a nivel global cerca de 2,5 millones de casos de hepatitis infecciosa, que resultaron en 25.000 casos fatales y en un número similar de incapacidades por daño hepático, con un impacto económico cercano a 10 billones de dólares anuales, sin mencionar epidemias de cólera $^{9}$. En el mundo, el total de enfermedades asociadas 
a la mala calidad del agua ha sido reportado en numerosos casos, de los cuales predominan el cólera, la fiebre tifoidea y la enfermedad diarreica aguda ${ }^{10}$.

La norma chilena de calidad del agua (NCh 409/1) con respecto al agua destinada para consumo humano establece que: "Todas las muestras que se analicen deben estar exentas de Escherichia coli". La norma establece que se acepta la presencia de $\mathrm{Ct}$ en una concentración menor o igual a 5 ufc o número más probable $\mathrm{NMP} / \mathrm{mL}$, siendo también aplicable para el agua de consumo animal ${ }^{11}$.

Por otra parte, la contaminación potencial del agua superficial y subterránea depende de factores como la estructura del suelo, los flujos de agua y la sobrevivencia de las bacterias en la superficie del suelo ${ }^{12}$. Variables como el pH, temperatura, conductividad, concentración de nitrógeno $(\mathrm{N})$ y fósforo $(\mathrm{P})$ afectan la dinámica de las poblaciones microbianas en el agua. Entre los parámetros químicos para evaluar la calidad del agua destacan la presencia de N y P; se estima que casi la mitad de ellos proviene de actividades agrícolas ${ }^{13}$. Uno de los problemas relacionados con altas concentraciones de nitrato en el agua es la formación de meta-hemoglobina, que produce falta de oxigenación en la sangre, proceso que se observa en infantes y fetos en gestación ${ }^{14}$. Sin embargo, las amenazas en la salud humana por los elevados niveles del nitrato en agua potable y productos alimenticios son todavía polémicas.

El impacto de los sistemas agrícolas-ganaderos ha sido ampliamente estudiado, debido al importante rol que tiene esta actividad en la contaminación del suelo, agua y atmósfera. Sin embargo, a medida que avanzan los sistemas productivos y el desarrollo de la actividad agropecuaria sigue en expansión, se hace imprescindible sumar esfuerzos con el fin de implementar medidas que permitan abarcar de una forma eficiente el problema de la contaminación del agua subterránea, lo que repercute en la salud humana. En base a lo expuesto se planteó investigar si las faenas agrícolas-ganaderas, con aplicación de purines y fertilizantes inorgánicos, provocan un impacto negativo en la calidad bacteriológica y físico-química del agua de consumo en áreas rurales, especialmente en periodos de alta actividad productiva en el centro-sur de Chile.

\section{Material y Método}

El área de estudio corresponde a un predio destinado a actividad agrícola y ganadera, ubicado en Pelchuquín, comuna de San José de la Mariquina, Provincia de Valdivia $\left(39^{\circ} 35^{`} \mathrm{~S}-73^{\circ} 05^{`} \mathrm{O}\right)$. El predio tiene una superficie de 600 hectáreas, consta de un plantel de 900 vacunos, de los cuales, 300 corresponden a vacas lecheras semiestabuladas. La mayor superficie del predio esta destinada a praderas para la alimentación del ganado y cultivos estacionales (maíz y nabos forrajeros), que son fertilizados con una mezcla de urea y súper fosfato triple $(230 \mathrm{~kg}$ de mezcla ha $^{-1}$ año $\left.{ }^{-1}\right)$ y purines en cantidades variables (11 $\mathrm{L} / \mathrm{m}^{2}$ ). El suelo corresponde a un andisol y el clima es de tipo templado cálido, con una precipitación anual de 1700 $\mathrm{mm}$ y la temperatura media anual de $10,8^{\circ} \mathrm{C}$.

Análisis microbiológico. La recolección de muestras de agua se realizó mensualmente (julio de 2008 a junio de 2009), en cada uno de cinco puntos de muestreo por triplicado (en total 15 muestras de agua/mes). Dos de los puntos de muestreo corresponden a agua superficial, colectadas al inicio $\left(39^{\circ} 35^{\prime} 1,7^{\prime \prime} \mathrm{S}-73^{\circ} 0422,1^{\prime \prime} \mathrm{O}\right) \mathrm{y}$ final $\left(39^{\circ} 35^{\prime} 47,5^{\prime \prime} \mathrm{S}-73^{\circ} 05^{\prime} 13,6^{\prime \prime} \mathrm{O}\right)$ de un estero que atraviesa el predio, el estero mide de ancho en su inicio $1,8 \mathrm{~m}$ y en su final $6 \mathrm{~m}$. Los restantes puntos de muestreo corresponden a agua subterránea extraída de pozos; $(\mathrm{PB})$ : primer pozo $\left(39^{\circ} 35^{\prime} 26,2^{\prime \prime} \mathrm{S}-73^{\circ} 04^{\prime} 22,1^{\prime \prime} \mathrm{O}\right)$ tiene agua destinada al consumo humano, $6 \mathrm{~m}$ de profundidad y se ubica a $20 \mathrm{~m}$ de una casa habitación. (PL): segundo pozo $\left(39^{\circ} 35^{\prime} 21,3^{\prime \prime} \mathrm{S}-73^{\circ} 04^{\prime} 22,1^{\prime \prime} \mathrm{O}\right)$, con agua destinada al consumo animal y lavado de material de lechería, de $6 \mathrm{~m}$ de profundidad y ubicado a 10 metros de la lechería. (PP): tercer pozo $\left(39^{\circ} 35^{\prime} 23,3^{\prime \prime} \mathrm{S}-73^{\circ} 04^{\prime}\right.$ $\left.21,5^{\prime \prime} \mathrm{O}\right)$, de 70 metros profundidad destinado abastecer al PL en periodos de escasez de agua, este pozo se ubica en el centro de la pradera. Todas las muestras de agua se colectaron en botellas estériles de capacidad de 1L. El agua de los esteros se recolectó manualmente a 0,5 m de la orilla y a $0,5 \mathrm{~m}$ de profundidad, el agua de los pozos fue recolectada desde la red (cañería) que trabaja con una bomba de vacío, para ello se abrió la llave respectiva, se dejó escurrir el agua durante dos minutos y luego se recolectó la muestra de agua en la botella respectiva. Cabe señalar que ninguna de las fuentes de agua experimenta tratamiento de cloración o de otro estilo. Todas las muestras fueron depositadas en una nevera con unidades refrigerantes y fueron llevadas a la Universidad Austral de Chile, para realizar los análisis microbiológicos y química del agua dentro de 2 y 24 horas, respectivamente.

A cada una de las muestras de agua se le determinó la presencia de Ct y E. coli de acuerdo al método confirmativo de determinación simultánea de Ct y E. coli QuantiTray. De la muestra de agua, se vertieron $100 \mathrm{~mL}$ en un matraz estéril de $250 \mathrm{~mL}$, posteriormente se adicionó el contenido del medio de cultivo Quanti-Tray. El matraz se agitó hasta que el contenido se disolvió por completo. A continuación, el contenido del matraz se depositó en un dispositivo Colilert Quanti-Tray estéril, el cual consta de 98 celdas, (49 grandes y 49 pequeñas). El dispositivo fue sellado herméticamente en una selladora térmica a $50^{\circ} \mathrm{C}$. Los dispositivos sellados fueron incubados a $35^{\circ} \mathrm{C}$ por $24 \mathrm{~h}$. Cualitativamente para las $\mathrm{Ct}$ se consideró como positivo las celdillas que presentaron color amarillo, para la determinación de $E$. coli se expusieron las celdillas a 
luz ultravioleta y aquellas que emitían fluorescencia se consideraron como positivas. También se determinó el NMP de Ct y E. coli, para ello se contó el número de celdillas grandes y pequeñas positivas, a continuación se buscaron los valores en una tabla y donde se intersectan ambos valores, aparece un número que indica el NMP de Ct o E. coli/100 $\mathrm{mL}$ de agua.

Análisis físico-químico del agua. A cada una de las muestras de agua se le midió la temperatura in situ, con un termómetro de mercurio, cuyos valores son expresados en grados Celsius. En el laboratorio se determinó el pH y conductividad individualmente a cada muestra de agua, con un $\mathrm{pH}$-metro y un conductivímetro de sobremesa. Los resultados de conductividad fueron expresados en $\mathrm{uS} / \mathrm{cm}$.

Determinación de fósforo total: se depositaron $50 \mathrm{~mL}$ de la muestra de agua en un matraz y se le agregaron $5 \mathrm{~mL}$ de solución oxidante $10 \%$; luego el matraz que contenía la solución se selló herméticamente y se llevó a autoclave a $121^{\circ} \mathrm{C}$ por 30 minutos. Posteriormente las muestras se dejaron enfriar y se les adicionó $3 \mathrm{~mL}$ de ácido ascórbico al 7\% más $3 \mathrm{~mL}$ de solución mixta al 25\%. La mezcla se dejó reposar 20 minutos y luego se leyó la absorbancia en un espectrofotómetro a $890 \mathrm{~nm}$. Los resultados se expresaron en $\mathrm{mg} \mathrm{P}-\mathrm{PO}_{4} / \mathrm{mL}$ de agua.
Determinación de nitrato $\left(\mathrm{N}-\mathrm{NO}_{3}\right)$ : se depositaron 25 $\mathrm{mL}$ de la muestra de agua en un matraz y se le agregaron $2 \mathrm{~mL}$ de reactivo colorante (ácido fosfórico $85 \% 100 \mathrm{~mL}$; sulfanilamida 10 g., N-1 naftil-etileldiamida 1 g., agua desionizada $1000 \mathrm{~mL}$ ), se homogenizó manualmente la mezcla y luego se leyó a $543 \mathrm{~nm}$. Los resultados se expresaron en $\mathrm{mg} \mathrm{N}-\mathrm{NO}_{3} / \mathrm{mL}$ de agua.

Análisis estadístico. A los resultados biológicos ( $\mathrm{Cf}$ y E. coli) y físico-químicos de cada mes, se les determinó el promedio. Con el fin de evaluar diferencia estadísticas en la calidad bacteriológica del agua, entre los distintos puntos de muestreo y en el tiempo, se hizo un análisis de varianza (Anova, $\mathrm{p}<0,05$ ), con sus respectivas pruebas de homocestacidad y normalidad. Se realizaron correlaciones (Pearson) entre la abundancia de poblaciones versus los parámetros físico-químicos $(\mathrm{p}<0,05)$. Todos los análisis se realizaron con el programa Statistica 6.0.

\section{Resultados}

En la Figura 1, se observa que en el agua superficial, el mayor NMP de $\mathrm{Ct} / 100 \mathrm{~mL} \mathrm{H}_{2} \mathrm{O}\left(3743 \mathrm{NMPCt}=\log _{10} 3,6\right)$ se determinó en el agua del estero salida (ES) en mayo y el menor valor $\left(162 \mathrm{NMP} \mathrm{Ct}=\log _{10} 2,2\right)$ en el estero de

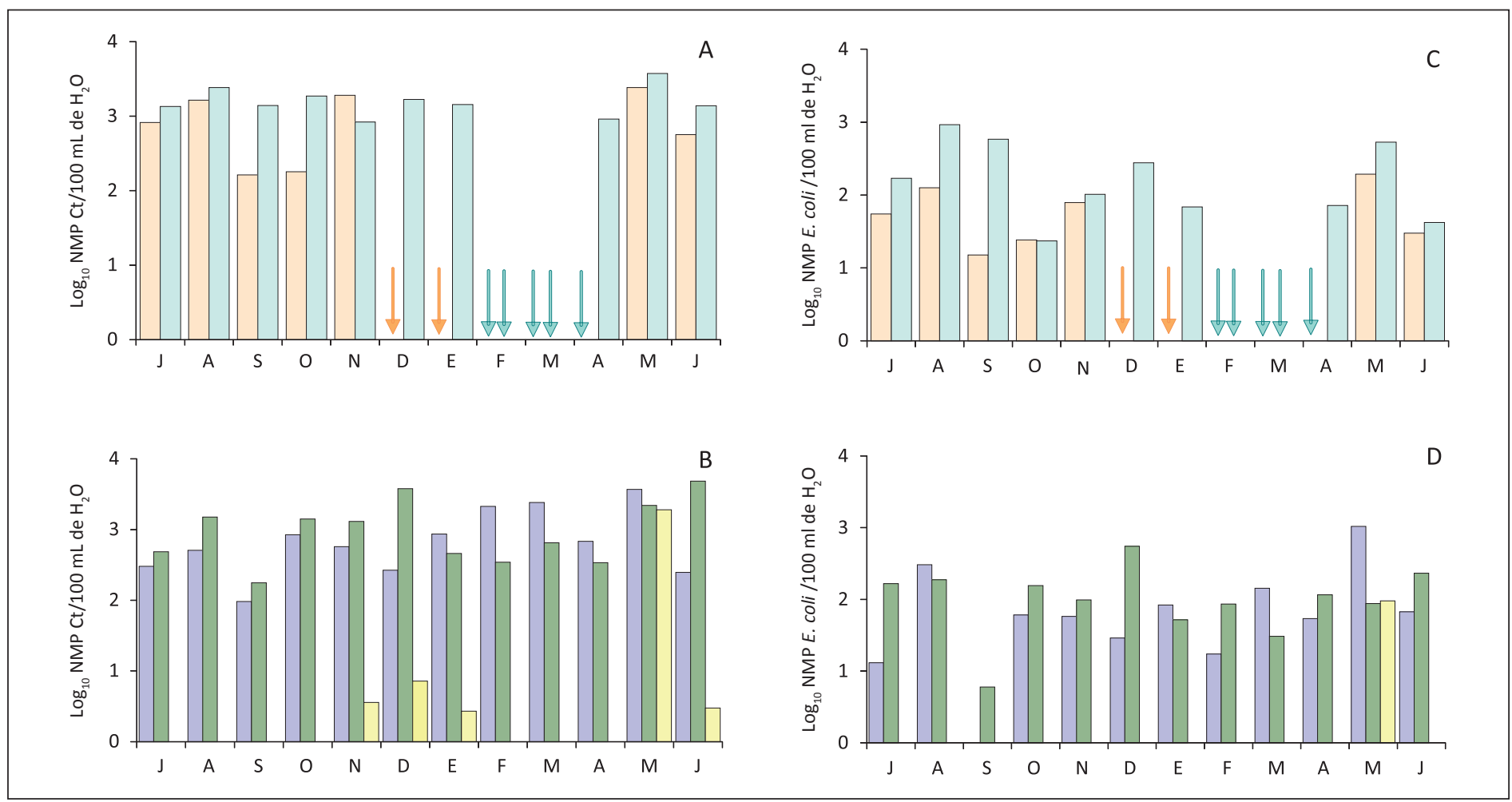

Figura 1. Número más probable (NMP/100 mL de agua) de: $A-B=$ coliformes totales (Ct) y C-D = Escherichia coli (E. coli), en cinco fuentes de agua de un predio agrícolaganadero del centro- sur de Chile. Estero origen $(\square$ EO). Estero salida ( $\square$ ES). Pozo bebida ( $\square$ PB). Pozo lechería ( $\square$ PL) y Pozo profundo ( $\square$ PP). Las flechas indican meses sin muestras de agua. 
origen (EO) en septiembre del 2008. Por su parte, el mayor NMP de E. coli/100 mL $\mathrm{H}_{2} \mathrm{O}$, se determinó en ES $(924$ $\left.\mathrm{NMPCf}=\log _{10} 3,0\right)$ en agosto y el menor en EO (15 NMP E. coli $\left.=\log _{10} 1,2\right)$ en septiembre 2008. En los pozos, el mayor NMP de $\mathrm{Ct} / 100 \mathrm{~mL} \mathrm{H}_{2} \mathrm{O}$, se determinó en el agua del pozo de lechería (PL), (4839 NMP Ct $\left.=\log _{10} 3,7\right)$ en junio de 2009, y los menores valores se determinaron en el agua del pozo profundo (PP) (0 NMP Ct) entre julioagosto del 2008 y febrero-abril del 2009. Por su parte, el mayor NMP de E. coli $/ 100 \mathrm{~mL} \mathrm{H}_{2} \mathrm{O}$, se determinó en el agua PB (1041 NMP E. coli $\left.=\log _{10} 3,0\right)$ en mayo del 2009 y los menores en el agua del PP (0 NMP E. coli) durante todo el periodo de muestreo, excepto mayo de 2009. En la Figura 1, se observa que en algunos periodos de muestreo de aguas superficiales (diciembre 2008 hasta abril 2009 en EO y febrero-marzo del 2009 en ES), las fuentes se encontraron sin agua y por lo tanto, el NMP de Cf o $E$. coli $/ 100 \mathrm{~mL} \mathrm{H}_{2} \mathrm{O}$ fue cero (sin registro).

Para las Ct en los esteros, el test de Tukey mostró diferencia estadísticamente significativa entre los sitios EO y ES y los meses, principalmente en septiembre y octubre para EO presentó en promedio 2,21 y 2,25 $\log _{10} \mathrm{NMP}$ $\mathrm{Ct} / 100 \mathrm{~mL}$ agua, respectivamente, siendo menores a los valores determinados para los otros meses. Con respecto a los pozos, PP no presentó Ct de julio a octubre y de febrero a abril. Para E. coli en los esteros, el test de Tukey mostró diferencia estadísticamente significativa entre EO y ES los menores valores se registraron en septiembre y octubre con 15 y $24 \log _{10}$ NMP E. coli/100 mL agua, respectivamente. Mientras que en los pozos se observó diferencias estadísticamente significativas entre PP con PB y PL, en PP durante todo el periodo de estudio no se observo presencia de E.coli excepto el mes de mayo donde se determinaron $2 \log _{10} \mathrm{NMP}$ E. coli $/ 100 \mathrm{~mL}$ agua.

La Figura 2 muestra los valores determinados de temperatura y precipitación. La precipitación más alta $(517 \mathrm{~mm})$ se determinó en agosto del 2008 y la menor (9,4 mm) en marzo del 2009. El análisis de varianza (Anova) no determinó diferencias significativas entre la precipitación por estaciones ( $\mathrm{f}=3,79 \mathrm{p}=0,05)$. La temperatura presentó un rango entre 13 y $18^{\circ} \mathrm{C}$. La mayor temperatura $\left(18^{\circ} \mathrm{C}\right)$ fue determinada en el agua de PL, diciembre del 2008 y las menores temperaturas $\left(13^{\circ} \mathrm{C}\right)$, se determinaron en el agua de los pozos y ES en junio del 2009. Para los esteros el test de Tukey mostró diferencias estadísticamente significativas entre EO y ES y entre los meses. En los pozos la diferencia significativa se presentó entre PB y PL y entre los meses.

En lo que respecta al $\mathrm{P}$ (Tabla 1), los valores promedios variaron entre 45,3 a 313,2 mg P-PO $/ \mathrm{L}$. En los esteros la mayor concentración se determinó en ES (68 mg P-PO $/ \mathrm{L}$ ) en enero del 2009 y la menor en EO (42 mg $\left.\mathrm{PO}_{4} / \mathrm{L}\right)$ en los meses de septiembre del 2008 y junio del 2009. En los pozos el valor más alto se determinó en PP (378 mg $\mathrm{PO}_{4} / \mathrm{L}$ ) en julio del 2008 y el más bajo en PB (44 mg P$\mathrm{PO}_{4} / \mathrm{L}$ ) en septiembre del 2008. En los esteros el test de Tukey mostró diferencias estadísticamente significativas entre EO y ES y entre los meses principalmente en febrero y marzo del 2009. En los pozos no se observó diferencia entre PP, PB y PL, mientras que en los meses se observó diferencia principalmente en mayo del 2009. Referente al $\mathrm{N}$ los valores promedios variaron entre 11,0 y 91,1 mg N-NO $/$ L. En los esteros la mayor concentración se determinó en EO (47 mg N-NO$/ \mathrm{L}$ ) en julio del 2009 y la menor se determinó en ES $\left(1,5 \mathrm{mg} \mathrm{N}-\mathrm{NO}_{3} / \mathrm{L}\right)$ en enero del 2009, presentando diferencia estadísticamente significativa. En los pozos el valor más alto se determinó en PP (182,1 mg N-NO $/ \mathrm{L})$ mayo del 2009 y el más bajo

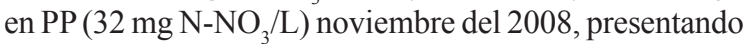
diferencia estadísticamente significativa.

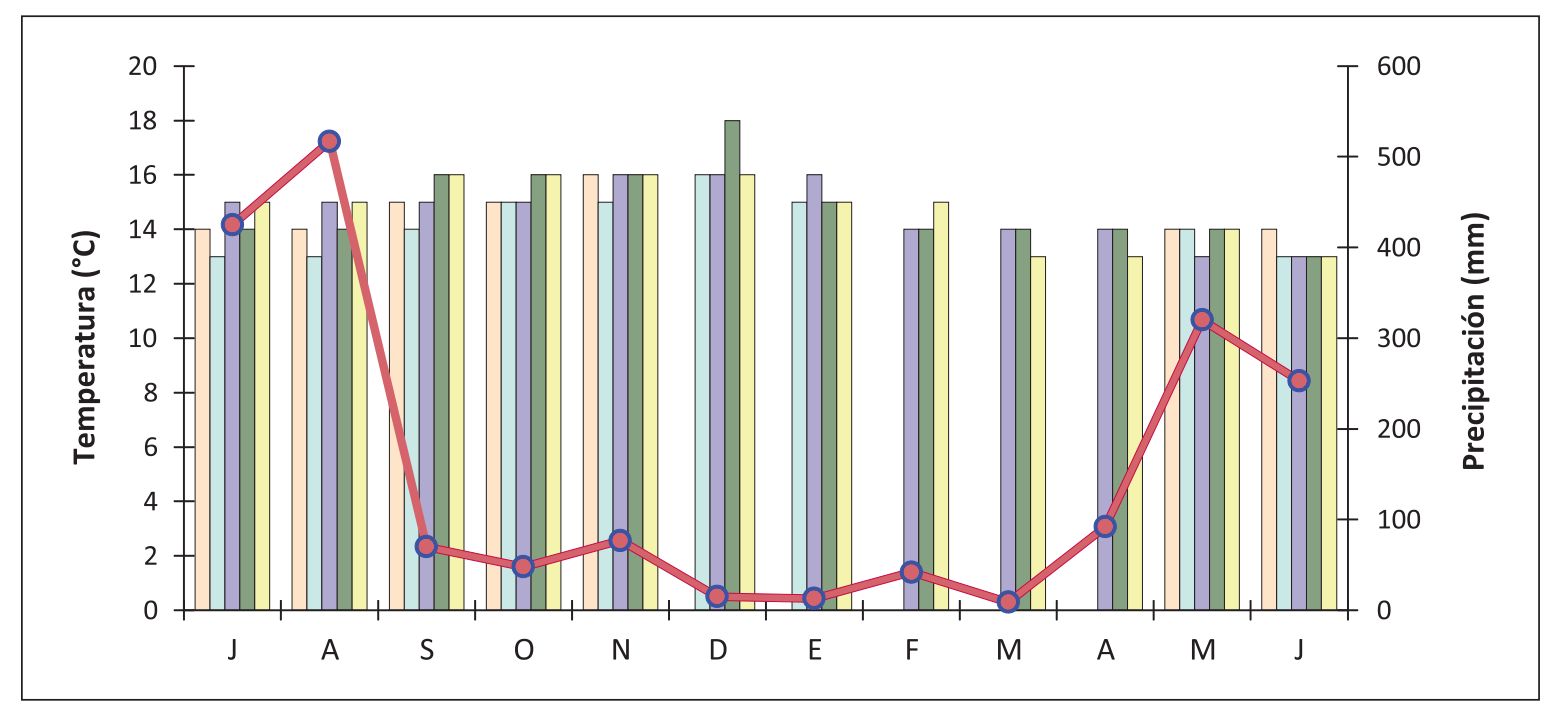

Rev Chilena Infectol 2012; 29 (6): 628-634
Figura 2. Temperatura $\left({ }^{\circ} \mathrm{C}\right)$ promedio del agua y precipitaciones, en cinco fuentes de agua de un predio agrícola y ganadero del Centro- Sur de Chile. Estero origen ( $\square$ EO). Estero salida ( $\square$ ES). Pozo bebida ( $\square$ PB). Pozo lechería ( $\square$ PL) y Pozo profundo ( $\square$ PP). (Valores promedios de 3 réplicas por sitio). La línea roja corresponde a la precipitación. 
Tabla 1. Valores promedios anuales de parámetros físico-químicos determinados en agua de esteros y pozos, de un predio agrícola-ganadero del Centro-Sur de Chile

\begin{tabular}{|c|c|c|c|c|c|c|}
\hline \multirow{2}{*}{\multicolumn{2}{|c|}{ Parámetros físico-químicos }} & \multicolumn{2}{|c|}{ Esteros } & \multicolumn{3}{|c|}{ Pozos } \\
\hline & & EO & ES & PB & PL & PP \\
\hline P & $\left(\mathrm{mg} \mathrm{P}-\mathrm{PO}_{4} / \mathrm{L}\right.$ de $\left.\mathrm{H}_{2} \mathrm{O}\right)$ & 45,3 & 58,8 & 70,5 & 152,1 & 313,2 \\
\hline N & $\left(\mathrm{mg} \mathrm{N}-\mathrm{NO}_{3} / \mathrm{L}\right.$ de $\left.\mathrm{H}_{2} \mathrm{O}\right)$ & 22,5 & 11,0 & 45,0 & 90,0 & 91,9 \\
\hline \multicolumn{2}{|c|}{$\mathrm{pH}$} & 7,86 & 8,20 & 8,20 & 7,87 & 9,54 \\
\hline \multicolumn{2}{|c|}{ Conductividad (uS/cm) } & 61,2 & 68,3 & 86,3 & 99,0 & 159,4 \\
\hline
\end{tabular}

EO: Estero origen. ES: Estero salida. PB: Pozo bebida. PL: Pozo lechería. PP: Pozo profundo.

Tabla 2. Correlaciones entre coliformes totales, E. coli, parámetros físico-químicos y variables microclimáticas (temperatura y precipitación) determinados en agua de esteros y pozos, de un predio agrícola-ganadero del Centro-Sur de Chile

\begin{tabular}{|cccccccc} 
& Sitio & pH & Conductividad & Fósforo & Nitrato & Temperatura & Precipitación \\
Ct & EO & n.s & 0,31 & n.s & 0,18 & n.s & n.s \\
& ES & $-0,27$ & $-0,06$ & 0,22 & 0,20 & n.s & 0,55 \\
& PB & $-0,53$ & n.s & 0,43 & 0,52 & n.s & $-0,03$ \\
& PL & $-0,27$ & 0,04 & $-0,26$ & 0,61 & 0,09 & 0,16 \\
& PP & n.s & n.s & n.s & 0,82 & $-0,23$ & 0,29 \\
& EO & 0,27 & 0,22 & n.s & 0,19 & 0,27 & 0,23 \\
& ES & 0,40 & $-0,34$ & $-0,35$ & 0,25 & $-0,26$ & n.s \\
& PB & $-0,37$ & 0,27 & $-0,08$ & 0,38 & $-0,44$ & 0,42 \\
& PL & 0,17 & 0,15 & $-0,03$ & 0,73 & 0,48 & 0,06 \\
& PP & n.s & n.s & n.s & 0,41 & $-0,24$ & 0,29 \\
\hline
\end{tabular}

Ct: Coliformes totales. EO: Estero origen. ES: Estero salida. PB: Pozo bebida. PL: Pozo lechería PP: Pozo profundo. n.s = valores no significativos climáticos del agua (Tabla 2), E. coli presentó una mayor correlación positiva con la conductividad, $\mathrm{pH}$, nitrato $\mathrm{y}$ precipitación, mientras que se estableció una relación negativa con el $\mathrm{P}$ y la temperatura.

\section{Discusión}

El máximo promedio anual de $\mathrm{Ct}$ y $E$. coli en las aguas superficiales se determinó en el ES, atribuido a la mayor exposición que tiene esta fuente $y$, por ende, al ingreso de materia orgánica, excrementos, tierra; incluso el agua del ES es usada para el lavado de maquinaria con desechos de lechería. Además, el agua del ES al atravesar el predio agrícola-ganadero produce lixiviación de las praderas arrastrando minerales, purines y fecas de los vacunos y otros animales. Los valores máximos de Ct y E. coli determinados en el agua de los esteros son menores a los registrados en el estero Piduco (Talca) el 2001, que variaron entre $137,823,500$ y $4,905 \mathrm{Ct} / 100 \mathrm{~mL}$ de agua y 30,000 a 3,000 E. coli (coliformes fecales) $/ 100 \mathrm{~mL}$ de agua (15). Por otra parte, las $\mathrm{Ct}$ superan a las determinadas en el estero Penco (2.400 Ct/100 mL de agua) y estero Lirquén ( $2.400 \mathrm{Ct} / 100 \mathrm{~mL}$ de agua) de Concepción, en lo que respecta a $E$. coli su número promedio supera al determinado en el estero Penco (292.8 E. coli/100 mL de agua), pero es menor al promedio determinado para el estero Lirquén $(1.818,8 \mathrm{E} \text {. coli/100 mL de agua })^{16}$.

En cuanto a la calidad microbiológica del agua del PB y PL, en la mayoría de los meses superaron ampliamente los valores máximos establecidos por la norma chilena de calidad del agua ${ }^{11}$. Posibles causas, pueden ser, la cercanía a lugares destinados a patios de alimentación y bebida del ganado, sitios de pasturas con una intensiva carga animal, pozos purineros o fosas sépticas, observándose un incremento y una fuerte relación entre estas fuentes potenciales de contaminación y el número de coliformes en el agua ${ }^{17}$. En el caso del PL, se encuentra cercano a la sala de ordeña y al lugar de alimentación del ganado; además contiguo se encuentra el pozo purinero, al cual constantemente son vertidas las excretas de los vacunos. Esto es importante especialmente bajo eventos de lluvias, que hacen que los desechos depositados aquí por efectos técnicos de capacidad de almacenamiento sean esparcidos en las praderas más cercanas al sitio de acopio, haciendo del área donde se encuentra el PL una zona altamente saturada con estiércol y restos de comida, los cuales fácilmente pueden escurrir por la pendiente del sitio hacia el pozo de agua de consumo.

En el caso del PB presenta altos valores de Ct y E. coli, esto se podría explicar por los problemas de construcción y ubicación, pues no presenta un emboquillado de cemento, tampoco una tapa adecuada que selle herméticamente el pozo y se encuentra cercano a una fosa séptica de la cual pueden escurrir desechos. 
El PP durante gran parte del estudio no presentó Ct y E. coli, exceptuando el mes de mayo. Esto se podría explicar por su lejanía de fuentes de contaminación, por presentar una mejor construcción y una mayor profundidad (70 m), lo que brinda una mayor protección ante la posibilidad de ser contaminado. Los valores positivos de contaminación microbiológica presente en algunos meses, podrían estar dados por hechos puntuales, como son la fertilización con purines por aspersión y eventos de lluvia, en el período de siembra.

Desde el punto de vista epidemiológico, los purines pueden contener un amplio rango de microorganismos patógenos tales como Salmonella spp., Campylobacter spp., Cryptosporidium y virus entéricos que pueden causar enfermedades en humanos ${ }^{17}$.

Al comparar los resultados con estudios realizados en sitios con alta actividad agrícola ganadera en Uruguay, entre 1996 y 1998 muestran que, de un total de 355 pozos analizados en zonas rurales se encontraba contaminado el $87 \%$, con Ct y $60 \%$ con E. coli. Además en una granja avícola en Argentina, se determinó que 54\% de los pozos presentaban contaminación con Ct y $16 \%$ con E. coli ${ }^{18}$.

Por su parte, en la ciudad de Ibadan en Nigeria, donde $77,1 \%$ de la población depende de agua de pozo para el uso doméstico, en una encuesta epidemiológica aplicada a 350 residentes, se determinó que la fiebre tifoidea presentó una alta incidencia $(39,3 \%)$, seguido de la disentería bacilar y el cólera. Estas enfermedades se relacionan al consumo de agua de pozos que presentaban contaminación fecal, pues las coliformes variaron entre 1 x $10^{3}$ y 18 x $10^{3} \mathrm{ufc} / \mathrm{mL}$ de agua ${ }^{19}$. En otro estudio en el distrito de Shunyi de Beijing, China, se analizó la calidad del agua de 434 pozos de auto-apoyo, tan sólo $65,4 \%$ de los pozos eran aptos para proveer de agua, el resto tenía exceso de: Fe; Mn; F; As; turbidez y coliformes, los autores del trabajo señalan que el agua debería desinfectarse en forma regular, para poder ser utilizadas por la población y evitar enfermedades causadas por bacterias y por elementos químicos como el $\mathrm{As}^{20}$. Como se señaló en la introducción, en Chile $40 \%$ del agua para consumo humano corresponde a agua subterránea, alcanzando a $80 \%$ en áreas rurales, donde los pozos profundos son la única fuente de agua de bebida para consumo humano y animal. En este estudio, los resultados indican una contaminación fecal del agua de los pozos analizados, que de acuerdo a la norma chilena de calidad del agua (NCh 409/1) no sería apta para el consumo humano, pudiendo ocurrir lo señalado en los estudios de Ibadan o Beijing.

Determinamos que el $\mathrm{P}$ presenta valores relativamente altos pero inferiores al estudio realizados por Nissen y cols. ${ }^{21}$, donde los valores de $\mathrm{P}$ en el agua de pozos profundos variaron entre 0,05 y $0,63 \mathrm{mg} / \mathrm{L}$. Al respecto, estudios muestran que un incremento en el P presente en los sedimentos, ejercería una importante influencia en la geoquímica del arsénico, ya que el $\mathrm{P}$ compite por los sitios de absorción favoreciendo la liberación de As a la columna de agua ${ }^{22}$. Por otra parte, en el agua de los pozos se determinaron altos niveles de $\mathrm{N}-\mathrm{NO}_{3}$, sobre todo en $\mathrm{PL}$ (90,0 mg N-NO $\left./ \mathrm{L} \mathrm{de}_{2} \mathrm{O}\right)$ y PP $\left(91,9 \mathrm{mg} \mathrm{N}-\mathrm{NO}_{3} / \mathrm{L}\right.$ de $\mathrm{H}_{2} \mathrm{O}$ ), estos valores superan los de la norma NCh901/1 para agua potable, que establece como máximo $50 \mathrm{mg} \mathrm{N}$ $\mathrm{NO}_{3} / \mathrm{L}^{23}$ y los de la Agencia para la Protección del Medio Ambiente Norteaméricana (EPA) cuyo limite es de $10 \mathrm{mg}$ $\mathrm{N}-\mathrm{NO}_{3} / \mathrm{L}$, e indica que cantidades superiores inducen a los microorganismos del estómago a la formación de metahemoglobina. Además los nitratos, también por acción de los microorganismos del estómago pueden formar nitrosaminas y nitrosamidas, compuestos potencialmente cancerígenos $^{24}$.

Por último, en Chile se llevó a cabo una investigación en una cuenca rural, cuya principal fuente de agua es subterránea, se analizaron 42 pozos, en todos se detectaron Ct, E. coli y Enterococcus spp., señalando que los pozos han sido contaminados con materia fecal humana y animal. Los autores indican como probables causas de la contaminación, el fácil acceso de animales domésticos a los pozos, el material de revestimiento permeable y el escurrimiento de fecas por la precipitación local, que ha influido directamente en la concentración de bacterias en los pozos ${ }^{25}$.

En base a los antecedentes entregados se puede concluir que las distintas fuentes de agua estudiadas presentan niveles de contaminación fecal y exceso de nitrógeno $\left(\mathrm{N}-\mathrm{NO}_{3}\right)$, que superan la norma chilena de calidad del agua (NCh 409/1) y que pueden ser dañinos para la salud humana.

Agradecimientos. Al proyecto FONDECYT 1085081 por el apoyo económico y al personal del área agrícola -ganadera, por las facilidades otorgadas para el estudio.

\section{Resumen}

Introducción: Parte importante del agua para consumo humano corresponde a agua subterránea, alcanzando un $80 \%$ en áreas rurales de Chile, donde los pozos profundos son la única fuente de agua de bebida para consumo humano y animal. Objetivo: Estudiar la calidad microbiológica del agua de un predio agrícola-ganadero en la provincia de Valdivia $\left(40^{\circ} \mathrm{S}\right)$, Chile, y su posible impacto en la salud humana. Material y Método: Muestras de agua fueron recolectadas mensualmente (2008-2009), a la entrada y salida de un estero que atraviesa el predio y en agua de pozos usadas para consumo humano y animal. Las especies coliformes totales $(\mathrm{Ct})$ y Escherichia coli se determinaron por el método confirmativo Quanti-Tray, se evaluaron variables físico-químicas del agua y climáticas 
del sitio. Resultados: En las muestras de agua del estero y pozos el número más probable de $\mathrm{Ct}$ y E. coli sobrepasaron la norma chilena de calidad del agua (NCh 409/1) para consumo humano. Conclusión: Estos resultados muestran la necesidad de regular el impacto ambiental de la actividad agrícola-ganadera y monitorear el agua de bebida para cumplir con los estándares mínimos de protección de la salud.

\section{Referencias bibliográficas}

1.- Godoy R, Oyarzún C, Gerding V. Precipitation chemistry in deciduos and evergreen Nothofagus forests in southern Chile under low-deposition climate. Basic Appl Ecology 2001; 2: 65-72.

2.- Oyarzún C, Godoy R, Leiva S. Depositación atmosférica de nitrógeno en un transecto valle longitudinal-cordillera de Los Andes, centro-sur de Chile. Rev Chil Hist Nat 2002; 75: 233-43.

3.- Salazar F, Dumont J, Santana M, Pain B, Chadwick D, Owen, E. Prospección del manejo y utilización de efluentes de lecherías en el sur de Chile. Arch Med Vet 2003; 35: 215-25.

4.- González M, López D, Gatica C. Effects of soiluse on deep groundwater quality in Southern Chile. J Environ Pollut 2004; 2: 240-52.

5.- Organización Mundial de la Salud. Guías para la calidad del agua potable. Primer apéndice a la tercera edición. Volumen 1. Recomendaciones. Organización Mundial de la Salud, Genève 27, Suiza 2006; 393 pp.

6.- Bordalo A, Onrassami R, Dechsakulwatana C. Survival of faecal indicator bacteria in tropical estuarine waters (Bangpakong River, Thailand). J Appl Microbiol 2002; 93: 864-71.

7.- Brooks G F, Butle J S, Morse S A. Microbiología Médica de Jawetz, Melnick y Adelbers. Ed El Manual Moderno, S. A de C. V (Mexico) 18 a Edición. 2005, Sección III, p 145-351 y Sección VII, pp 701-731.

8.- LeJeune J, Besser T, Merrill N, Rice D, Hancock, D. Livestock drinking water microbiology and the factors influencing the quality of drinking water offered to cattle. Dairy Sci 2001; 84: 1856-62.
9.- United Nations Environment Programme (UNEP). Urgent action need to protect the marine environmem. UNEP. 2001. http//www. unep.org/ (consultado 25/10/2009).

10.- Ojeda L, Arias R. Informe Nacional sobre la gestión de agua en Colombia (Recursos hídricos, agua potable y saneamiento). Ministerio de Medio Ambiente, Santafé de Bogota. 2000; 137 p.

11.- Chile, Instituto Chileno de Normalización (INN). Norma chilena NCh 409/1. Agua potable parte 1: requisitos. Instituto Nacional de Normalización. Chile. 2005, p 14.

12.- González M, López D, Gatica C. Effects of soiluse on deep groundwater quality in Southern Chile. J Environ Pollut 2004; 21 (3): 240-52.

13.- Werner W. Agriculture and diffuse nutrient inputs into surface waters, seminar on agriculture and aquaculture. Environ North Seas 1991; 6: 113-4.

14.- Townsend A, Howarth R, Bazzaz F, Booth M, Cleveland C, Collinge S, et al. Human health effects of a changing global nitrogen cycle. Frontiers in Ecol. and Environ 2003; 1: 240-6.

15.- Conejeros E. Calidad del agua del estero Piduco (Talca VII Región): un análisis basado en datos existentes. Theoria 2003; 12: 43-54.

16.- Mondaca M, Campos V, Luengo E, Reyes V, Acuña N, Rivas N, et al. Evaluar la calidad microbiológica de las aguas de la comuna de Penco. Proyecto Explora ED/04/069 Universidad de Concepción 2007; 27-31.

17.- Pell A. Manure and microbes: Public and animal health problem? J. Dairy Sci 1997; 80: $2673-81$
18.- Perdomo C, Casanova O, Ciganda V. Contaminación de aguas subterráneas con nitratos y coliformes en el litoral sudeste del Uruguay. Agrociencia 2001; 5: 10-22.

19.- Oguntoke O, Aboderin O, Bankole A. Association of water-borne diseases morbidity pattern and water quality in parts of Ibadan city, Nigeria. Tanzania J. Health Res 2009; 11: 189-95.

20.- Wang R, Liang H, Zhen G, Zhen D, Huang X, Sun $\mathrm{X}$, et al. Hygienic investigation on rural-owned well-water at Shunyi district and application in improving water-quality in the countryside. Modern Prev Med 2009; 36: 3423-5.

21.- Nissen M, Garay M, Aguilera A, Valenzuela E. Calidad de aguas subterráneas de la Décima Región de Chile. Agro Sur 2000; 28: 25-39.

22.- Rubinos D, Barral M, Ruiz B, Ruiz M, Rial M, Álvarez M. Phosphate and arsenate retention in sediments of the Anllons River (northwest Spain). Water Sci. and Technol 2003; 48: 159-66.

23.- Chile, Instituto Chileno de Normalización (INN). Norma chilena NCh 409/1.Agua potable parte 1: requisitos. Instituto Nacional de Normalización. Chile. 2005, p 5.

24.- USA. Environmental Protection Agency. integrated risk information system. Nitrate (CASRN 14797-55-8) http:/www.epa.gov/iris/ subst/0076.htm (consultado 2/1/2012).

25.- Valenzuela M, Lagos B, Claret M, Mondaca M, Pérez C, Parra O. Fecal contamination of groundwater in a small rural dryland watershed in Central Chile. Chilean J Agric Res 2009; 69: $235-43$. 